\title{
Patient engagement in research: a systematic review
}

\author{
Juan Pablo Domecq ${ }^{1,2,5}$, Gabriela Prutsky ${ }^{1,2,5}$, Tarig Elraiyah' ${ }^{1,5}$, Zhen Wang ${ }^{1,5,6}$, Mohammed Nabhan ${ }^{1,5}$, \\ Nathan Shippee ${ }^{1,5,6}$, Juan Pablo Brito ${ }^{1,4,5}$, Kasey Boehmer ${ }^{1,5}$, Rim Hasan ${ }^{1,5,8}$, Belal Firwana ${ }^{1,5,8}$, Patricia Erwin 1,7, \\ David Eton ${ }^{1,5,6}$, Jeff Sloan ${ }^{1,5,6}$, Victor Montori ${ }^{1,2,4,5,6}$, Noor Asi ${ }^{1,5}$, Abd Moain Abu Dabrh ${ }^{1,5}$ \\ and Mohammad Hassan Murad ${ }^{1,3,5,6^{*}}$
}

\begin{abstract}
Background: A compelling ethical rationale supports patient engagement in healthcare research. It is also assumed that patient engagement will lead to research findings that are more pertinent to patients' concerns and dilemmas. However; it is unclear how to best conduct this process. In this systematic review we aimed to answer 4 key questions: what are the best ways to identify patient representatives? How to engage them in designing and conducting research? What are the observed benefits of patient engagement? What are the harms and barriers of patient engagement?
\end{abstract}

Methods: We searched MEDLINE, EMBASE, Psyclnfo, Cochrane, EBSCO, CINAHL, SCOPUS, Web of Science, Business Search Premier, Academic Search Premier and Google Scholar. Included studies were published in English, of any size or design that described engaging patients or their surrogates in research design. We conducted an environmental scan of the grey literature and consulted with experts and patients. Data were analyzed using a non-quantitative, meta-narrative approach.

Results: We included 142 studies that described a spectrum of engagement. In general, engagement was feasible in most settings and most commonly done in the beginning of research (agenda setting and protocol development) and less commonly during the execution and translation of research. We found no comparative analytic studies to recommend a particular method. Patient engagement increased study enrollment rates and aided researchers in securing funding, designing study protocols and choosing relevant outcomes. The most commonly cited challenges were related to logistics (extra time and funding needed for engagement) and to an overarching worry of a tokenistic engagement.

Conclusions: Patient engagement in healthcare research is likely feasible in many settings. However, this engagement comes at a cost and can become tokenistic. Research dedicated to identifying the best methods to achieve engagement is lacking and clearly needed.

Keywords: Systematic review, Patient, Engagement, Patient centered outcomes research

\section{Background}

The role of patient in research ranges from a passive one (patient is a data point) to an active one (patient is a researcher). The active participation in research (or patient engagement in research) can potentially lead to improvement in the credibility of results (higher rates of

\footnotetext{
*Correspondence: murad.mohammad@mayo.edu

'Knowledge and Evaluation Research Unit, Mayo Clinic, Rochester, MN, USA ${ }^{3}$ Division of Preventive, Occupational and Aerospace Medicine, Mayo Clinic, Rochester, MN, USA

Full list of author information is available at the end of the article
}

enrollment and retention) and in their direct applicability to patients (by asking pertinent questions about patientimportant outcomes). Also, there is an overarching ethical mandate for patient participation in research as a manifestation of the "democratization" of the research process [1-3]. Patient engagement in the planning and execution of research could also improve its translation into clinical practice [4]. In all, there is growing consensus about the crucial role of patient involvement in research, which may improve the value of healthcare research. 
In the United Kingdom, involvement of stakeholders in social and health care policy has been well recognized since 1996. The British National Institute of Health recognized that individual and community stakeholders determine important aspects of health care services and research, and the project INVOLVE was established to achieve this engagement $[5,6]$. In the United States, the Patient Centered Outcomes Research Institute (PCORI) was established in 2010 and placed great importance on the engagement of patients and other stakeholders in the research process [7].

Previous systematic reviews have described various aspects of the engagement process [8,9]. However, it remains unclear who to engage or when, or how to perform this task [8-10]. Therefore, PCORI commissioned a systematic review that aims at synthesizing the existing evidence about patient engagement in research with the goal of helping researchers in designing and conducting meaningful patient engagement in healthcare research. In this systematic review we aimed to answer 4 key questions: what are the best ways to identify patient representatives? How to engage them in designing and conducting research? What are the observed benefits of patient engagement? What are the harms and barriers of patient engagement?

\section{Methods}

This systematic review is conducted based on a priori established protocol (Additional file 1) and is reported according to the PRISMA statement [11]. The PRISMA checklist is available in Additional file 2.

\section{Eligibility criteria}

We included all original studies of any design, size, or patient population published in the English language in which patients or their surrogates provided feedback, had input, or took part in the design, conduct and dissemination of research. Systematic reviews were also included to supplement the findings from original studies. Other non-original studies (non-systematic literature reviews, comments, opinions, letters and editorials etc.) were excluded. In general, we sought studies in which patients were actively engaged in designing research. Participation in surveys was only considered to be research engagement when the main purpose of the survey was to obtain patients' values and preferences that relate to research prioritization or research design.

\section{Patient advisory group}

The protocol of this systematic review was developed after consultation with patients from the Patient Advisory Council [12]. This is a group of volunteer patients from Rochester, Minnesota who have contributed to the design of multiple studies over the last 10 years. The group helped in developing the questions and outcomes of the review and advised on terminology. They also reviewed the results and provided feedback on the presentation of findings, usefulness and applicability.

\section{Search}

\section{Electronic search}

An expert librarian (PJE) collaborated with a methodologist (MHM) to develop the search strategy. We searched biomedical electronic databases [PubMed/Ovid MEDLINE, Ovid EMBASE, Ovid PsycInfo, Ovid Cochrane, EBSCO CINAHL, SCOPUS, Web of Science (multidisciplinary scientific content), Business Search Premier, Academic Search Premier and Google Scholar (communications, marketing, public opinion, and business literature that incorporate non-healthcare resources)] from their inception through November 2011 (Additional file 1).

To identify additional candidate studies we reviewed reference lists from eligible studies and conducted additional MEDLINE searches using the PubMed "related articles" feature for eligible studies. We used SciSearch for publications that cited eligible studies to supplement the database search.

\section{Environmental scan}

We complemented the database search with an environmental scan and a manual search. The environmental scan includes searching the Internet using various search engines for recent and ongoing activities, initiatives, white papers and websites to identify key players and trends in the field. It helps provide content from grey (unpublished) literature and from fields other than medicine. We also searched the scientific search engines Scirus and Sciverse, which contain scientific journal content, scientists' homepages, courseware, pre-print server material, patents, and institutional repository and website information. In addition, we contacted experts in the field to identify other relevant documents (e.g., dissertations, scientific reports). The environmental scan, as expected, identified some of the published literature already included in the systematic review; overlapping references were excluded.

\section{Study selection}

We collated initial references in citation files (using Endnote software), removed duplicates, and screened titles and abstracts against eligibility criteria using DistillerSR software (Evidence Partners Incorporated, Ottawa, Canada). Studies were reviewed in duplicate until almost perfect agreement (Kappa >0.80) [13] was achieved, after reviewing 200 potentially includible references. Disagreements in the initial screening were automatically included. Potentially eligible studies were then reviewed in full text 
following a similar procedure. Disagreements in full-text screening were reconciled by discussion, consensus, or arbitration by study principal investigator (MHM). We exclusively used electronic file formats (Portable Document Format/PDF), in reference management software to reduce costs and paper use.

\section{Data extraction}

Data were extracted from included studies using a standardized form developed based on the protocol. We tested this form using a small sample $(\mathrm{n}=10)$ of randomly selected studies, from which all reviewers extracted data. The first author (JPD) evaluated each extraction form and compared the extracted data between reviewers and discussed discrepancies with them.

Data extracted from each study included: study description (e.g., demographics of participants and research setting), methods used to select patients (defined as a patient, surrogate, caregiver, community member, or other stakeholder informing research), measures set in place to enhance the validity or completeness of identifying patients (e.g., selecting methods -convenience, random, volunteer, training level for the task), measures of validity or accuracy of the information or input given by participants (the patient's voice, e.g., validation of the patient reported outcome measurement, congruence of patient's voice with other stakeholders), description of methods used to implement/incorporate the patient's voice in research, and any reported outcomes of patient engagement. We also captured authors' recommendations about the methods to be used for eliciting the patient's voice and facilitating patient engagement.

\section{Analysis}

The nature of the question of this systematic review along with the lack of standard approach across existing studies prevented a quantitative meta-analysis. Instead, data extracted from the included studies were analyzed using a meta-narrative approach [14]. This approach was developed as a pragmatic solution to study topics that have been differently conceptualized and studied by different groups of researchers [15]. The approach starts by a standard systematic review with explicit inclusion and exclusion criteria. Analysis follows a framework that defines the key questions. The included studies are evaluated until saturation for discrete themes and trends that can be mapped to outcomes. Differences in studies settings and characteristics can be used to explain differences in results (heterogeneity) [16].

The analytic framework of this systematic review is depicted in Figure 1 showing the 4 key questions of interest. Following this approach, patient engagement experiences reported in the literature were classified into categories based on how patients were selected and engaged. Then, we attempted to map each category to an engagement outcome corresponding to the 4 key questions to allow inference.

We also categorized the research engagement from each study into three different research phases proposed for patient engagement [17]:

1) Preparatory phase (agenda setting, prioritization of research topics and funding).

2) Execution phase (study design \& procedures, study recruitment, data collection, and data analysis).

\section{Patient engagement in healthcare research}

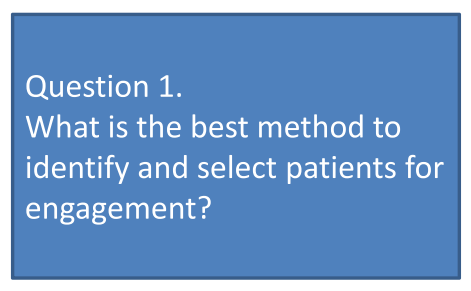

Question 2.

How to best engage patients?

-Timing (stage of research)

-Methods of engagement
Question 3 .

What are the benefits of

engagement (changes in

study design, higher

enrollment, etc.)

Question 4.

What are the harms/barriers?

Figure 1 Analytical framework. 
3) Translation phase (dissemination, implementation, and evaluation).

Results are presented following the sequence of the 4 key questions as depicted in the framework (Figure 1) with illustrative examples.

\section{Results}

\section{Search and selection results}

Overall we identified 5,562 possibly relevant citations, of which 142 met the eligibility criteria and were included. Studies reported a spectrum of engagement. Studies described patient engagement in research preparation phase (35), execution phase (90) and translation phase (52). Some studies contributed to our understanding of more than one phase.

In terms of the 4 key questions of this review, we found 121 studies that contributed to our understanding of the first question regarding patient selection; 45 studies that contributed to our understanding of the second question regarding engagement methods; 43 studies that reported on the third question regarding engagement outcomes; and 36 studies that reported on the fourth question regarding barriers and challenges of engagement. Many studies contributed to our understanding of more than one of the 4 key questions.

The study selection process is described in Figure 2. The studies included 8 systematic reviews, 7 randomized trials and 24 observational studies; the remaining majority (103) consisted of qualitative studies. Additional file 3: Tables S1 and S2 describe the characteristics of the systematic reviews and original studies. Due to the qualitative component of our research question and the heterogeneity of studies design, the methodological quality of the included studies was evaluated using selected items from the list proposed by the Critical Appraisal Skills Programme (CASP) [18,19]. The studies overall had a few limitations particularly in the areas of patient selection and data synthesis (Additional file 3: Table S3). Additional file 3: Table S4 shows the list of initiatives and patient organizations engaging patients in research identified by the environmental scan.

\section{Overview of existing systematic reviews}

The literature search identified 8 relevant systematic reviews that addressed various aspects of patient engagement and spanned across the 4 key questions. A Cochrane systematic review by Nilsen et al. [8] reported that engaging patients in the research process may lead to an output (report) that is more readable and understandable by other patients. The authors concluded that there are insufficient data to evaluate the impact of patient engagement. Nevertheless, they found that the engagement was feasible in most of their included randomized controlled trials.

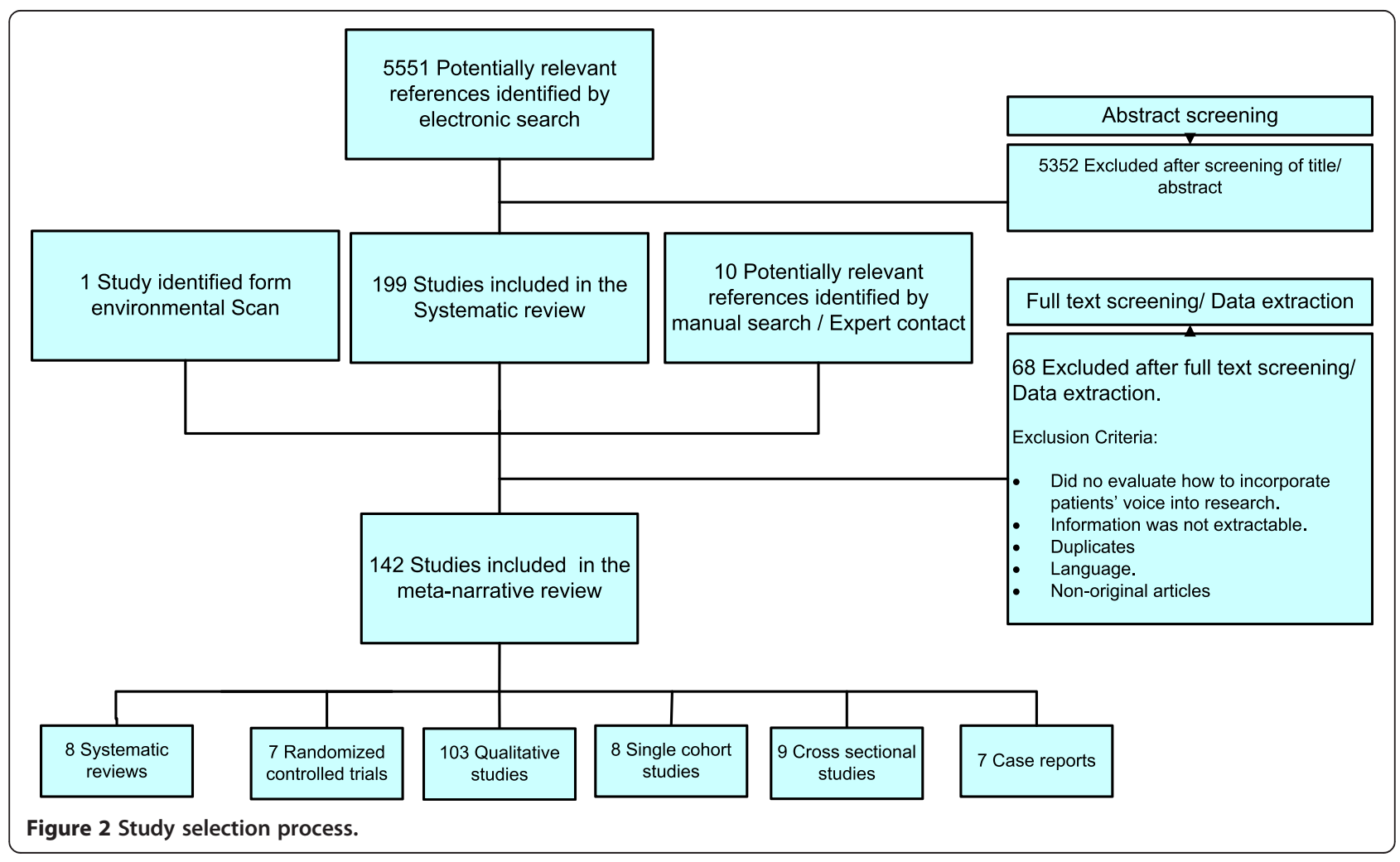


The systematic review by Mockford et al. [9] evaluated the impact of patient and public involvement in the UK National Health Service programs. The review summarized 42 studies and concluded that there is little evidence of any economic analysis of the costs involved, poor quality of reporting, minimal theoretical or conceptual underpinning, lack of measurement and evaluation; and overall weak evidence base to support patient and public engagement.

Brett et al. [1] examined the conceptualization, measurement, impact and outcomes of patient and public involvement in health and social care research. They concluded that there is an emerging and important evidence of the impact of patient engagement on health care research but with relatively little conceptualization and theoretical development in the field. They also described poor quality of reporting as a one of the most important barriers restricting our understanding of the impact of patient engagement in research.

Boote et al. [20] reviewed published case examples of public involvement in primary research design and reported that group meetings were the most common method used to engage the public and that most patient contributions were in the areas of review of consent procedures and patient information sheets, outcome suggestion, and recommendations on participants recruitment.

Three systematic reviews by Legare et al., Oliver et al., and Stewart et al., summarized collectively over 250 studies in which patient engagement was conducted $[10,21,22]$. The three reviews reported similar key findings and focused on describing topics and stages of research most amenable to engagement and common methods of engagements (e.g., meetings, workshops and focus groups) that should be tailored to the topic of research at hand. These reviews also highlighted challenges and barriers to engagement $[10,21,22]$.

Lastly, Hussain-Gambles [23] focused on engaging South Asian patients in designing clinical trials and reported on the factors that motivate patients to participate as well as deterrents. The review highlighted that there are more similarities than differences in attitudes towards clinical trials between the South Asian and the general population. The main findings and conclusions of the 8 systematic reviews are reported in Additional file 2: Table S3.

\section{Key questions}

\section{Question 1. What are the best methods to identify patients} for engagement?

In general, most of the studies described convenience sampling as the method to identify patients (or representatives/ surrogates) for engagement in research. Therefore, patients attending clinics or other patient care facilities were approached and asked to participate. Patients also volunteered in a response to advertisements or Internet postings. Very few studies randomly selected patient representatives. For example, researchers in the Netherland randomly selected patients with asthma and chronic obstructive pulmonary disease from the entire pool of the Netherlands Asthma Foundation and engaged these patients in consultation to define their health research priorities [24]. This engagement resulted in additional prioritization of other research topics that were not covered by current Dutch research programs. Researchers in Canada randomly selected from patients attending outpatient cancer therapy to evaluate their attitudes, motivations and barriers to participation in clinical trials [25]. Murad et al. asked a random sample of patients with diabetes about their preferences for future trials in diabetes in terms of design (pragmatic vs. explanatory trial design) and outcomes (surrogate vs. hard endpoints) [26].

We found no comparative analytic studies to provide evidence supporting a particular method to identify or select patients for engagement in research.

\section{Question 2. What are the best methods to engage patients?}

Studies described a variety of methods that were used to engage patients, the most common of which were focus groups, interviews, surveys and the most active form of engagement which is serving on a study board or advisory council and attending regular meetings with researchers (as in active participatory research studies and community based participatory research). For example, Swartz et al. conducted a randomized controlled trial to test the effectiveness of a pollutant and allergen control strategy on the symptoms of childhood asthma [27]. In the early stages of the study, researchers engaged several stakeholders (2 school principals, a pastor, a nun, 2 community association presidents, a social worker, a parent of a child with asthma and a health care worker who had previously served in the same community). The engaged persons took the role of an advisory board and a partner in research. They helped develop study protocol, recruitment procedures and selected outcomes. They also educated study personnel about the community and attended presentations and meetings to obtain necessary approvals for the study. They subsequently even helped with execution of study intervention (measured pollutant levels in demolition sites). Crowe et al. described the engagement of Hispanic farm workers and their families in Yakima Valley, Washington, in every stage of a study including study concept and design, data collection, data analysis and interpretation, conclusions, and dissemination of results [28]. Thirteen community members and stakeholders met 
regularly throughout the duration of the study providing input and making decisions about research execution. The engaged persons also presented alongside researchers to the larger community during Town Hall meetings.

We found no comparative analytic studies to provide evidence supporting a particular method of engagement. The different methods used for engagement are depicted in Figure 3 and are stratified according to research phase.

\section{Question 3. What are the observed benefits of patient engagement?}

Several studies reported that engaging patients in research improves patient enrollment and decrease attrition [10,27,29-31]. For instance, Edwards et al. [30] conducted a randomized controlled trial of osteopathy in children with cerebral palsy compared to a control group that only received standard therapy. They demonstrated that engaging parents in study design led to higher enrollment and retention rates. Likewise, Swartz et al. [27] conducted a randomized controlled trial in inner-city children with asthma comparing environmental control education, allergen-proof encasements, pest extermination, and an air filter to a control group that only received standard therapy. The used a community based participatory research approach, which achieved a high enrollment and retention rates, $86 \%$ and $70 \%$ respectively. They also reported that engagement helped in dissemination to the extent that their reporting was more meaningful and understandable for participants and the community.

\section{Question 4. What are the harms and barriers of patient engagement?}

While most studies reported mainly positive effects of engagement, [1] a smaller number described potential harms or adverse effects of engaging patients. These harms mainly related to patient frustration with the lengthy process that involved training, transportation, attendance, etc. [31,32]. In terms of barriers and challenges, studies cited logistics such as extra time needed to complete research, time constraints of patients and researchers, and incremental funding needed for patient engagement. Another common concern and an overarching worry of researchers and patients was that patient engagement may become tokenistic [33,34] (a false appearance of inclusiveness), resulting in a devaluated patients' input. Another potential challenge described was "scope creep"; a theoretical concern that engaging patients in the research may include irrelevant community concerns and issues, which would make the research unfeasible $[27,35]$.

Of the few studies that described potential solutions, the most commonly described were spending adequate time to build reciprocal relationships [17] (between patients and researcher), fostering mutual respect and developing clear expectations that are explicitly described and documented in study protocols. We found no comparative studies to provide supportive evidence for question 4 .

\section{Discussion}

This meta-narrative systematic review identified numerous heterogeneous studies in which patients or their surrogates (other patients) were successfully engaged in

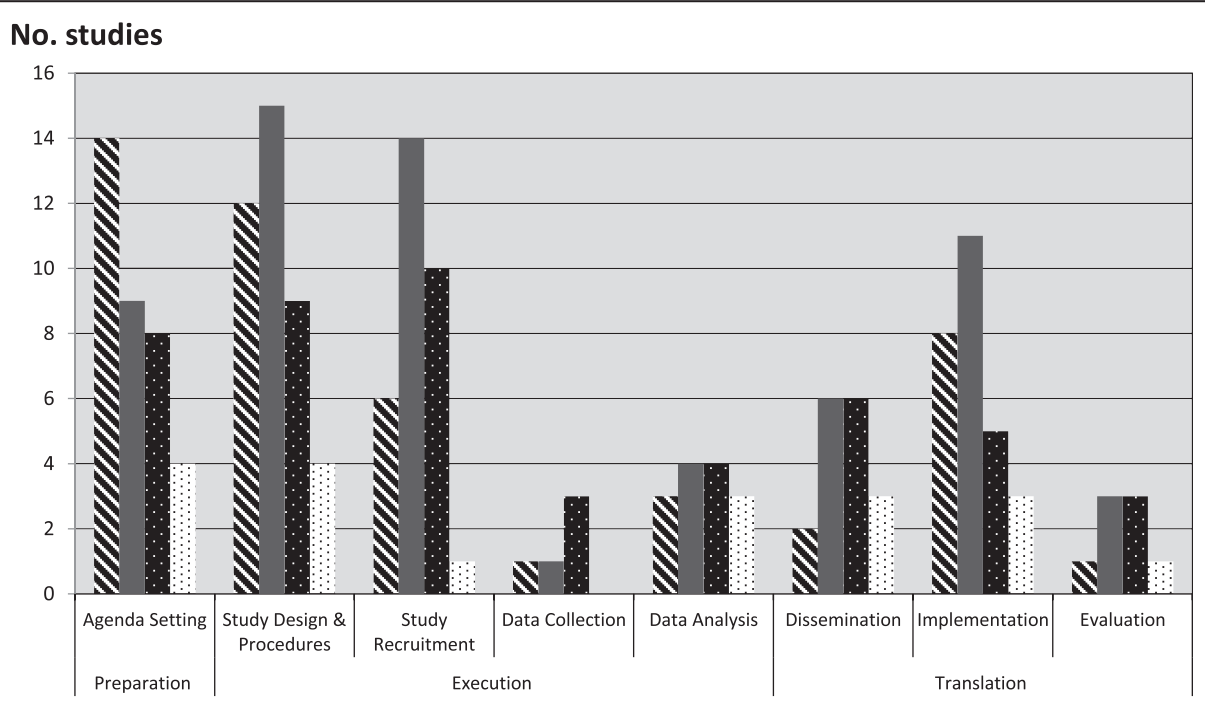

s Focus Group Interview Survey : Deliberation/Organizational participation

Figure 3 Methods and phases of engagement. 
the research process. The engagement was feasible in most of the published cases we found, but faced several challenges and barriers. Most studies claimed some benefits of this process; however, there were no comparative data to suggest best practices.

\section{Strengths and limitations}

Heterogeneity of study populations, methods, and outcomes constitute limitations of this synthesis. Publication and reporting biases might have impacted the conclusions of this report and their impact cannot be estimated. Our search may have missed studies in which patient engagement was performed due to the lack of uniform reporting or indexing methods of engagement. Evaluating the quality of patient engagement is challenging due to unavailability of validated scales and the limited data reported in the studies. We did not find the standard tools for assessing the methodological quality of the studies particularly useful because such assessment actually relates to the outcomes of the study and not to the outcome of patient engagement. Such assessment can be misleading as it is quite possible to have a study with low risk of bias for its primary and secondary outcomes that performed tokenistic or ineffective patient engagement.

Our results are consistent with other systematic reviews in the field $[1,8,9]$. The current review updates the evidence base to date and provides a contemporary look at patient engagement. Developed with active participation from patients, researchers, and the PCORI staff (as an external experts' advisory group that did not participate in conducting this SR), this systematic review takes priority in establishing the baseline starting point from which we need to advance the science of patient engagement in research. This review utilized a comprehensive and sensitive search strategy that spanned across multiple databases and was augmented by an environmental scan of non-peer-reviewed relevant sources to further capture related studies, web sites, and interest groups. Our application of an a priori protocol for selecting and appraising evidence reduces selection bias. The thematic analysis of this review sought to ensure presenting the evidence without over-interpreting its signals and silences, a key concern in this area.

\section{Practical implications}

At the present time we are unable to recommend best practices on the basis of comparative evidence. However, many methods are described in the literature with reported success. In terms of identifying patients for engagement, random sampling is the least biased way although considering that the number of patients chosen for engagement is very small, random sampling can fail. This approach is also challenging in rare diseases. Most of the included studies used volunteers which is a more practical method despite the potential for having a sample of patients that are not truly representative of the targeted population. Volunteers may be more educated and motivated and engage more effectively, yet they may have personal agendas. At the present time, we suggest that researchers choose their method of selection based on the availability of subjects and the research topic at hand.

Engaging patients in all research phases (preparatory, execution and translation) seems feasible in most cases. This was even demonstrated in populations and communities with high prevalence of social inequities (intellectual disparities, poverty, unemployment and illiteracy) traditionally considered difficult to reach [36-40]. The engagement process may improve the credibility of results and their applicability to the target population and may have an empowering effect on participants. Potential risks (harms and costs) for engaged patients should be balanced against the broad range of articulated potential benefits.

Future research in this field is greatly needed to demonstrate the value of patient engagement to researchers and funders. For example, patient engagement when conducting systematic reviews has been recommended by the Agency for Healthcare Research and Quality Evidence-based Practice Centers, the Institute of Medicine, the Cochrane Collaboration, and others [41]. Yet, there is no guidance on how to perform this engagement. A possible study in this field can randomize systematic reviewers to test three approaches, one without engaging patients, one with engaging patients with the condition being studied, and one with engaging patients with any condition (general patients). Qualitative studies can be embedded in most trials to evaluate different engagement strategies or engagement in different phases of the trials. Clinical practice guidelines are mostly done without patient engagement;[42] however this engagement is described by many as paramount and essential $[43,44]$. The impact of patient representation on guideline panels cane be studied using qualitative research methods to determine if their presence was tokenistic or meaningful. Lastly, we recommend that bibliographic databases use indexing terms that identify active patient engagement in research to facilitate future research in this field.

\section{Conclusions}

Patient engagement in healthcare research is likely feasible in many settings. However, this engagement comes at a cost and can become tokenistic. Research dedicated to identifying the best methods to achieve engagement is lacking and clearly needed. 


\section{Additional files}

\section{Additional file 1: Protocol and Search Strategy. Additional file 2: PRISMA 2009 checklist.}

Additional file 3: Table S1. Systematic reviews. Table S2. Original studies. Table S3. Quality assessment of the original studies. Table S4. Initiatives and patient organizations identified by the environmental scan.

\section{Competing interests}

The authors declare that they have no competing interests.

\section{Authors' contributions}

All listed authors significantly contributed to this project. Study protocol was developed by MHM, VMM, JPD and PJE. JPD, GP, TE, ZW, NA, AA, MN, JB, KB, $\mathrm{RH}$ and $\mathrm{BF}$ carried out study selection and data extraction. NS, DE and JS provided the content expertise for study design and participated in writing the discussion section of the manuscript. PJE is the reference librarian who conducted the literature search. VMM and MHM provided the methodological expertise and supervised the work. All authors read and approved the final manuscript.

\section{Acknowledgements}

We thank members of the Patient Advisory Group, Mayo Clinic, Rochester, for providing feedback at various stages of this project.

This systematic review was funded by the Patient-Centered Outcomes Research Institute (PCORI).

\section{Author details}

${ }^{1}$ Knowledge and Evaluation Research Unit, Mayo Clinic, Rochester, MN, USA ${ }^{2}$ Unidad de Conocimiento y Evidencia, Lima, Perú. ${ }^{3}$ Division of Preventive, Occupational and Aerospace Medicine, Mayo Clinic, Rochester, MN, USA. ${ }^{4}$ Division of Endocrinology, Diabetes, Metabolism, Nutrition, Mayo Clinic, Rochester, MN, USA. ${ }^{5}$ Center for the Science of Healthcare Delivery, Mayo Clinic, Rochester, Minnesota, USA. ${ }^{6}$ Department of Health Sciences Research, Mayo Clinic, Rochester, MN, USA. ${ }^{7}$ Mayo Clinic Libraries, Mayo Clinic, Rochester, MN, USA. ${ }^{8}$ Department of Internal Medicine, University of Missouri, Columbia, MO, USA

Received: 26 September 2013 Accepted: 20 February 2014

Published: 26 February 2014

\section{References}

1. Brett J, Staniszewska S, Mockford C, Seers K, Herron-Marx S, Bayliss H: The PIRICOM Study: A Systematic Review of the Conceptualisation, Measurement, Impact and Outcomes of Patients and Public Involvement in Health and Social Care Research. Warwick: University of Warwick; 2010. http://wrap.warwick.ac. uk/47235/.

2. Thompson J, Barber R, Ward PR, Boote JD, Cooper CL, Armitage CJ, Jones G: Health researchers' attitudes towards public involvement in health research. Health Expect 2009, 12(2):209-220.

3. Viswanathan M, Ammerman A, Eng E, Garlehner G, Lohr KN, Griffith D, Rhodes S, Samuel-Hodge C, Maty S, Lux L, Webb L, Sutton SF, Swinson T, Jackman A, Whitener L: Community-based participatory research: assessing the evidence. Evid Rep Technol Assess (Summ) 2004, 99:1-8.

4. Sullivan P, Goldmann D: The promise of comparative effectiveness research. JAMA 2011, 305(4):400-401.

5. National Institute for Health Research (INVOLVE project). [http://www.invo.org.uk/about-involve/]

6. Equity and excellence: liberating the NHS. [https://www.gov.uk government/uploads/system/uploads/attachment_data/file/213823/dh_ 117794.pdf]

7. Patient-Centered Outcomes Research Institute. [http://www.pcori.org/ research-we-support/pcor/]

8. Nilsen ES, Myrhaug HT, Johansen M, Oliver S, Oxman AD: Methods of consumer involvement in developing healthcare policy and research, clinical practice guidelines and patient information material. Cochrane Database Syst Rev 2006, 3:CD004563.
9. Mockford C, Staniszewska S, Griffiths F, Herron-Marx S: The impact of patient and public involvement on UK NHS health care: a systematic review. Int J Qual Health Care 2012, 24(1):28-38.

10. Oliver S, Clarke-Jones L, Rees R, Milne R, Buchanan P, Gabbay J, Gyte G, Oakley A, Stein K: Involving consumers in research and development agenda setting for the NHS: developing an evidence-based approach. Health Technol Assess 2004, 8(15):1-148.

11. Moher D, Liberati A, Tetzlaff J, Altman DG: Preferred reporting items for systematic reviews and meta-analyses: the PRISMA statement. Ann Intern Med 2009, 151(4):264-269. W264.

12. Patient Advisory Group: The wiser choices program of the Knowledge and Encounter Research Unit, Mayo Clinic. [http://shareddecisions. mayoclinic.org/stakeholders/diabetes-advisory-group/]

13. Viera AJ, Garrett JM: Understanding interobserver agreement: the kappa statistic. Fam Med 2005, 37(5):360-363.

14. Greenhalgh T, Robert G, Macfarlane F, Bate P, Kyriakidou O, Peacock R: Storylines of research in diffusion of innovation: a meta-narrative approach to systematic review. Soc Sci Med 2005, 61(2):417-430.

15. Wong G, Greenhalgh T, Westhorp G, Buckingham J, Pawson R: RAMESES publication standards: meta-narrative reviews. BMC Med 2013, 11:20

16. Tong A, Flemming K, McInnes E, Oliver S, Craig J: Enhancing transparency in reporting the synthesis of qualitative research: ENTREQ. BMC Med Res Methodol 2012, 12:181

17. Shippee ND, Domecq Garces JP, Prutsky Lopez GJ, Wang Z, Elraiyah TA, Nabhan M, Brito JP, Boehmer K, Hasan R, Firwana B, Erwin PJ, Montori VM, Murad $\mathrm{MH}$ : Patient and service user engagement in research: a systematic review and synthesized framework. Health Expect 2013. doi: 10.1111/hex.12090

18. Critical Appraisal Skills Programme (CASP). Making sense of evidence. 10 questions to help you make sense of qualitative research. England: Public Health Resource Unit. [http://www.casp-uk.net/wp-content/uploads/ 2011/11/CASP-Qualitative-Research-Checklist-31.05.13.pdf]

19. Campbell R, Pound P, Pope C, Britten N, Pill R, Morgan M, Donovan J: Evaluating meta-ethnography: a synthesis of qualitative research on lay experiences of diabetes and diabetes care. Soc Sci Med 2003 56(4):671-684

20. Boote J, Baird W, Beecroft C: Public involvement at the design stage of primary health research: a narrative review of case examples. Health Policy 2010, 95(1):10-23.

21. Stewart RJ, Caird J, Oliver K, Oliver S: Patients' and clinicians' research priorities. Health Technol Assess 2011, 14(4):439-448.

22. Legare F, Boivin A, van der Weijden T, Pakenham C, Burgers J, Legare J, St-Jacques S, Gagnon S: Patient and public involvement in clinical practice guidelines: a knowledge synthesis of existing programs. Med Decis Making 2011, 31(6):E45-E74.

23. Hussain-Gambles M, Leese B, Atkin K, Brown J, Mason S, Tovey P: Involving South Asian patients in clinical trials. 2004, 8((42):iii):1-109.

24. Caron-Flinterman JF, Broerse JE, Teerling J, Bunders JF: Patients' priorities concerning health research: the case of asthma and COPD research in the Netherlands. Health Expect 2005, 8(3):253-263.

25. Jones JM, Nyhof-Young J, Moric J, Friedman A, Wells W, Catton P: Identifying motivations and barriers to patient participation in clinical trials. J Cancer Educ 2006, 21(4):237-242.

26. Murad MH, Shah ND, Van Houten HK, Ziegenfuss JY, Deming JR, Beebe TJ, Smith SA, Guyatt GH, Montori VM: Individuals with diabetes preferred that future trials use patient-important outcomes and provide pragmatic inferences. J Clin Epidemiol 2011, 64(7):743-748.

27. Swartz LJ, Callahan KA, Butz AM, Rand CS, Kanchanaraksa S, Diette GB, Krishnan JA, Breysse PN, Buckley TJ, Mosley AM, Eggleston PA: Methods and issues in conducting a community-based environmental randomized trial. Environ Res 2004, 95(2):156-165.

28. Crowe JL, Keifer MC, Salazar MK: Striving to provide opportunities for farm worker community participation in research. J Agric Saf Health 2008, 14(2):205-219

29. Shagi C, Vallely A, Kasindi S, Chiduo B, Desmond N, Soteli S, Kavit N Vallely L, Lees $S$, Hayes $R$, Ross D: A model for community representation and participation in HIV prevention trials among women who engage in transactional sex in Africa. AIDS Care 2008, 20(9):1039-1049.

30. Edwards V, Wyatt K, Logan S, Britten N: Consulting parents about the design of a randomized controlled trial of osteopathy for children with cerebral palsy. Health Expect 2011, 14(4):429-438. 
31. Shah SG, Robinson I: Benefits of and barriers to involving users in medical device technology development and evaluation. Int I Technol Assess Health Care 2007, 23(1):131-137.

32. Oliver SR, Rees RW, Clarke-Jones L, Milne R, Oakley AR, Gabbay J, Stein K, Buchanan P, Gyte G: A multidimensional conceptual framework for analysing public involvement in health services research. 2008, 11(1):72-84

33. Minogue V, Girdlestone J: Building capacity for service user and carer involvement in research: the implications and impact of best research for best health. 2010, 23(4):422-435.

34. Smith YR, Johnson AM, Newman LA, Greene A, Johnson TRB, Rogers JL: Perceptions of clinical research participation among African American women. 2007, 16(3):423-428.

35. Decker M, Hemmerling A, Lankoande F: Women front and center: the opportunities of involving women in participatory health research worldwide. 2010, 19(11):2109-2114.

36. Stewart P, Shibasaki $S$, Anderson I, Pyett P, Dunbar T, Devitt J: Aboriginal and Torres Strait Islander participation in ethical review of health research. 2006, 30(3):291-292.

37. Evans S, Corley M, Corrie M, Costley K, Donald C: Evaluating services in partnership with older people: exploring the role of 'community researchers'. 2011, 15(1):26-33.

38. McLaughlin H: Involving young service users as co-researchers: possibilities, benefits and costs. Br J Soc Work 2006, 36(8):1395-1410.

39. Johnson K: No longer researching about us without us: a researcher's reflection on rights and inclusive research in Ireland. 2009, 37(4):250-256.

40. Walmsley J: Involving users with learning difficulties in health improvement: lessons from inclusive learning disability research. 2004, 11(1):54-64.

41. Kreis J, Puhan MA, Schunemann HJ, Dickersin K: Consumer involvement in systematic reviews of comparative effectiveness research. Health Expect 2013, 16(4):323-337.

42. Wyatt KD, Stuart LM, Brito JP, Carranza Leon B, Domecq JP, Prutsky GJ, Egginton JS, Calvin AD, Shah ND, Murad MH, Montori VM: Out of context: clinical practice guidelines and patients with multiple chronic conditions: a systematic review. Med Care 2013 [Epub ahead of print].

43. Montori VM, Brito JP, Murad MH: The optimal practice of evidence-based medicine: incorporating patient preferences in practice guidelines. JAMA 2013, 310(23):2503-2504.

44. Murad MH, Montori VM, Guyatt GH: Incorporating patient preferences in evidence-based medicine. JAMA 2008, 300(21):2483. author reply 2483-2484.

\section{Submit your next manuscript to BioMed Central and take full advantage of:}

- Convenient online submission

- Thorough peer review

- No space constraints or color figure charges

- Immediate publication on acceptance

- Inclusion in PubMed, CAS, Scopus and Google Scholar

- Research which is freely available for redistribution 